Rev. Elev. Méd. Vét. Poys Trop., 1966, 19, 4 (527-43).

\title{
Etude dans certaines conditions africaines de l'action antiparasitaire du Thiabendazole sur divers helminthes des animaux domestiques
}

\section{II. - Dromadaire}

\author{
por M. GRABER \\ Institut d'Elevage et de Médecine Vétérinaire des pays tropicaux \\ Laboratoire de Farcha-Fort-Lamy, République du Tchad
}

\begin{abstract}
RÉSUMÉ
L'auteur, à partir d'essais effectués au Tchad sur 85 dromadaires originaires du Kanem et du Batha, conclut que le Thiabendazole est inactif sur un grand nombre d'Anoplocepholidae de l'intestin, sur Schistosomo bovis, sur les Hydatides d'Echinococcus gronulosus, sur Buckleyuris globulosa et sur:Globidium cameli.

Contre les Nématodes gastro-intestinaux les plus fréquents, deux dosages peuvent être préconisés :

- 100 d̀ $150 \mathrm{mg} / \mathrm{kg}$ (produit pur). Cette dose est capable de détruire en totalité une association à base de Strongyloides papillosus, Trichostrangylus vitrinus, Trichostrongylus probolurus ef Impalaia nudicollis, tous Nématodes particulièrement dangereux pour le chameau.

- $300 \mathrm{mg} / \mathrm{kg}$ qui représentent pour le parasitologue la dose la plus polyvalente, car elle est capable d'agir à la fois sur les Helminthes précédents sur Haemoncus longistipes de la caillelte (à 85 p. 100) et sur CEsophagostomum columbianum du gros iniestin. Cette dose, au Tchad, doit être retenue de préférence et utilisée de mai à novembre au moment où le parasitisme gastro-intestinal du dromadaire est le plus abondant.

Le Thiabendazole est assez peu toxique pour le chameau, sauf si les animaux sont atteinls d'autres affections chroniques (Trypanosómiase ; abcès multiples ; pneumonies, etc...). II faut alors réduire de moitié la dose à'administrer, quitte à recommencer par la suite.

Après traitement, l'état général s'améliare ropidement, dans la mesure où l'animal est placé sur un pâturage convenable, de type plutôt arbustif. Le gain de poids va de $+6,8$ p. 100 à 11 p. 100 .

L'anihelminthique ne devra donc être employé chez le chameau qu'en prenant quelques précautions.
\end{abstract}

\section{INTRODUCTION}

Les Helminthiases du dromadaire constituent, avec la Gale et la Trypanosomiase, les trois affections les plus meurtrières de l'Elevage camelin du Tchad.
Le parasitisme - surtout gastro-intestinal est principalement à base de Nématodes (GRABER et Coll., 1967) : Strongyloides papillosus (WEDL, 1856), Trichostrongylus vitrinus (LOOSS, 1905). Trichostrongylus probolurus (RAILLIET, 1896). Nematodirus spothiger (RAILLIET, 1896), 
Impalaı nudicollis (MÖNNIG, 1931), Hoemoncus longistipes (RAILLIET et HENRY, 1909), Oesophagostomum (Proteracrum) columbianum (CURTICE, 1890) et Buckleyuris (= Trichuris) globulosa (VON LINSTOW, 1901).

Pour tenter de détruire ces Helminthes, divers auteurs ont employé la Phénothiazine à des doses variables : en Russie, de 20 à $60 \mathrm{~g}$ par tête (OZERSKAYA, 1953) et de 75 d̀ $500 \mathrm{~g}$ par tête (IVASKHINA, 1953). Au Soudan, STEWARD (1950) administre $600 \mathrm{mg} / \mathrm{kg}$ sans incident ef, au Niger FERRY (1961) $100 \mathrm{~g}$ par animal deux jours de suite.

Au Tchad, il avait été recommandé (GRABER, 1955) de distribuer deux fois à 24 heures d'intervalle deux doses de $110 \mathrm{~g}$ chacune pour les animaux les plus lourds et deux doses de $60 \mathrm{~g}$ pour les plus légers. Au bout d'une semaine, le nombre d'oufs au gramme de matière fécale dıminue de moitié et l'état général s'améliore.

Malheureusement, d'autres observations effectuées en fin de saison sèche 1956 (Rap. Ann. Ouaddai) ont montré, sur des chameaux très bas d'état et très parasités, que la Phénothiazine, aux doses globales de 220 ou de $120 \mathrm{~g}$, était capable de provoquer des accidents toxiques graves, voire mortels. Le dosage a été réduit à $160 \mathrm{~g}$ (2 fois $80 \mathrm{~g}$ ), ce qui semble nettement insuffisant, $200 \mathrm{~g}$ constituant d'après FERRY (1961) la limite inférieure d'efficacité de la Phénothiazine à l'égard des parasites gastrointestinaux du dromadaire.

Devant ces résultats décevants, il a paru intéressant de rechercher si le 2-(4'-Thiazolyl) benzimidazole ou Thiabendazole, dont l'action anthelminthique chez les bovins et les ovins est par alleurs bien connue (GRABER, 1965), pouvait se substituer à la Phénothiazine et, dans l'affirmative, quelles seraient les conditions d'utilisation chez le dromadaire tchadien.

\section{MATÉRIEL ET MÉTHODE}

\section{A. — LES ANIMAUX D'EXPÉRIENCE}

Au total, 85 dromadaires adultes pesant de 230 d̀ $440 \mathrm{~kg}$ ont été utilisés. Ils se répartissent àinsi :

Essais thérapeutiques proprement dits : 30 . Essais de toxicité : 3.
Essais sur le terrain : 27.

Témoins : 25 .

Les 58 animaux ayant servi aux opérations 1 , 2 et 4 étaient originaires du Kanem (OuestTchad) et du Batha (Centre-Tchad). Les témoins, venus des mêmes régions, ont été sacrifiés à la même époque que les animaux traités. Quant aux essais sur le terrain, ils ont été réalisés au Ouaddai (Est-Tchad) où les conditions, du point de vue parasitisme, se rapprochent de celles du Batha.

L'état d'entretien des animaux, dans l'ensemble était médiocre, plus de la moitié des chameaux présentant une anémie marquée, liée à la présence de nombreux Helminthes dans le tractus digestif.

La plupart des dromadaires hébergeaient un grand nombre de parasites (Tableau I) :

Dans 85 p. 100 des cas, ces parasites se trouvaient associés par 2 (23 p. 100), $3(10,4$ p. 100), 4 (20,8 p. 100$), 5$ (12,5 p. 100), 6 (25 p. 100) et $7(8,3$ p. 100) espèces différentes. Ces associations - surtout en matière de Nématodes - ont permis d'étudier la polyvalence éventuelle du Thiabendazole.

Les expériences ont été menées en cinq étapes de février 1965 à janvier 1966 (février-mars 1965 ; juin-juillet 1965 ; octobre-novembre 1965 ; décembre 1965 et janvier 1966). Compte tenu de ce que l'on sait de la biologie du parasite le plus commun, Haemoncus longistipes, cet étalement couvre :

- une période favorable au développement de ce Nématode (de juin à octobre). Le nambre moyen d'Haemoncus rencontré à l'autopsie de chaque animal est alors d'environ 1.300 (de 300 à 3.500 ) et, dans bien des cas, ils sont sexuellement immatures, tout en ayant atteint leur taille adulte. Il s'agit d'une première infestation, les conditions hygrométriques contribuant à la pullulation des larves d'Hoemoncus,

- une période moins favorable où les infestations, du falt de la sécheresse persistante, sont assez faibles et où le taux de parasitisme (de la mi-novembre à la mi-mai) est bien inférieur à ce qu'il est au début ou à la fin, de la saison des pluies (150 Hoemoncus longistipes en moyenne par tête). Beaucoup de parasites sont alors sexuellement mûrs. 
TABLEAU NOI

Nombre d'animaux atteints (

\begin{tabular}{|c|c|c|c|c|c|}
\hline \multirow{2}{*}{ Espèces en cause } & \multicolumn{2}{|c|}{ Kanem } & \multicolumn{2}{|c|}{ Batha } & \multirow[t]{2}{*}{ Total } \\
\hline & Traités & Témoing & Traités & Témoins & \\
\hline Schistosome bovis & 1 & - & 2 & 2 & 5 \\
\hline Woniezia expansa & 3 & - & 9 - & 1 & 13 \\
\hline Moniezia benedeni & 2 & - & 1 & - & 3 \\
\hline Thysaniezia ovilla & 3 & 1 & - & - & 4 \\
\hline Avitelitina centripunctata & - & 1 & 1 & - & 2 \\
\hline Avitellina woodlandi* & 2 & - & 1 & 1 & 4 \\
\hline St1lesia globipunctata & 4 & - & 5 & 2 & 11 \\
\hline Echinococeus polynorphus & 3 & - & 8 & 11 & 22 \\
\hline Strongyloides papillosus & 11 & 1 & 14 & 2 & 28 \\
\hline Desophagostomum columbianum & 7 & - & 18 & 2 & 27 \\
\hline Trichostroneylus vitrinus & & & & & \\
\hline Mrichostrongylug probolurug & 2 & - & 8 & 2 & 12 \\
\hline Impaleia nudicollua & & & & & \\
\hline Heamoncus longistipes & 12 & 1 & 17 & 24 & 54 \\
\hline Buckleyruris globulosa & 13 & 1 & 16 & 5 & 35 \\
\hline Globidium cameli & 6 & - & 5 & 1 & 12 \\
\hline
\end{tabular}

* Que Spassici (1951) considère comme une forale d'Avitellina centripunctata.

TABLBRAU NOII

Témoins.

Fóvrier $1965: 1$ (Kanem) - Juin-Juillet $1965: 22$ (Batha) - Novembre $1965: 2$ (Batha)

\begin{tabular}{|c|c|c|c|c|c|c|}
\hline \multirow{2}{*}{ Helminthes en cause } & \multicolumn{3}{|c|}{ Nombre de chaneaux } & \multicolumn{3}{|c|}{ Poids ou nombre de parasites } \\
\hline & Février & $J_{113 n}$ & Novembre & Févriler & Juin & Novembre \\
\hline $\begin{array}{l}\text { Schistosoma bovis } \\
\text { Moniezia expansa } \\
\text { Thysantezia ovilla } \\
\text { Aritelline woodlandi } \\
\text { Stilesia globipunctata } \\
\text { Echinococcus polynorphis } \\
\text { Strongyloldes papillosus } \\
\text { Oesophagostomum columbianum } \\
\text { Impalala nudicolilis } \\
\text { Trichostrongylus vitrinus } \\
\text { Trichostrongylus probalurus } \\
\text { Haemoneus longistipes } \\
\text { Buckleyurio globulosa } \\
\text { Globidium cameli }\end{array}$ & $\begin{array}{l}- \\
1 \\
\overline{-} \\
\overline{1} \\
- \\
- \\
1 \\
1 \\
-\end{array}$ & $\begin{array}{l}- \\
- \\
7 \\
9 \\
- \\
2 \\
- \\
22 \\
3 \\
-\end{array}$ & $\begin{array}{l}2 \\
1 \\
- \\
1 \\
1 \\
1 \\
2 \\
1 \\
2 \\
\\
1 \\
2 \\
1\end{array}$ & $\begin{array}{l}\overline{-} \\
\overline{10} \\
- \\
- \\
\overline{7} \\
- \\
- \\
78 \\
32 \\
-\end{array}$ & $\begin{array}{r}- \\
- \\
- \\
7 \\
= \\
20 \\
- \\
925 \\
13 \\
-\end{array}$ & $\begin{array}{c}5 \\
13,5 \\
6,5 \\
1 \\
1 \\
10 \\
25 \\
9 \\
197 \\
7 \\
-\end{array}$ \\
\hline
\end{tabular}

Cestodes : poids moyen en grammes

Nemetodes, Schistosames et hydatides : moyenne du nombre de parasites. 


\section{B. - MÉTHODE}

\section{Avant traitement.}

Chaque animal est mis en observation pendant 48 heures et des examens coproscopiques ef hématologiques sont pratiqués dans le but de situer l'importance exacte du parasitisme.

Des cultures d'œufs permettent de préciser la nature des Helminthes en cause. En fonction des renseignements ainsi obtenus, les lots sont constitués. Ils comprennent un tiers d'animaux très parasités, un tiers d'animaux moyennement parasités et un tiers d'animaux faiblement parasités.

\section{Traitement.}

Les chameaux ont été marqués et placés dans des stalles individuelles cimentées. Le Thiabendazole a été donné sans diète préalable «à la bouteille ».

\section{Après traitement.}

Sur chaque animal, il a été procédé durant 8 à 10 jours :

a) Au prélèvement des fèces trois fois dans la journée. Elles ont été broyées dans un mince filet d'eau ef soigneusement examinées, de manière à faire apparaître les Helminthes Cestodes et Nématodes - évacués après l'administration du médicament. Ceux-ci ont été pesés, comptés et déterminés.

b) A des examens coproscopiques journaliers par la méthode de sédimentation. Dans un lot, la comparaison entre les moyennes du nombre d'cuufs au gramme avant traitement, après traitement et le jour de l'autopsie permet déjà 'de se faire une idée, même approximative, de la valeur du médicament.

c) A des cultures d'œufs en boîte de Petri sur papier buvard humide. Arbitrairement, on dénombre chaque jour le nombre de larves $\mathrm{L}_{3}$ rencontrées dans une goutte de suspension aqueuse provenant des boîtes de Petri. Les cufs d'Hoemoncus longistipes mettent 4 à 7 jours, au laboratoire, pour atteindre le stade $L_{s}$.

Pour chaque lot, la comparaison entre la moyenne du nombre de larves avant ef après traitement complète, en général, les éléments d'appréciation fournis par l'examen coproscopique. Pour cerłains Nématodes digérés dans l'intestin après l'administration du Thiabendazole (Impalaia, Trichostrongylus, Strongylordes), la méthode est même absolument indispensable.

d) Autopsie.

Passé ce délai, les animaux sont sacrifiés. Les Helminthes demeurés en place sont récoltés: ce travail ne pose pas de problème pour les grands Cestodes, les Oesophagostomes adultes, les Trichures et les Hoemoncus de la caillette. La comparaison entre le nombre (ou le poids) de parasites expulsés et le nombre (ou le poids) de parasites encore présents dans l'intestin donne le pourcentage d'efficacité.

Lorsqu'il s'agit de petits Nématodes ou de Stilesia globipunctota, il importe de gratter la muqueuse duodénale sur une longueur de 30 à $50 \mathrm{~cm}$. L'examen au microscope du produit de raclage placé entre lame ef lamelle confirme ou non la présence d'Impalaia, de Strongyloides ou de scolex de Stilesia globipunctata. Pour ce dernier, la comparaison entre le nombre total de nodules et le nombre de scolex retrouvés donne le pouvoir antheiminthique du Thiabendazole à l'égard de ce Cestode.

Le protocole est donc très classique : il combine les méthodes habituellement employées dans les essais thérapeutiques intéressant les Cestodes et les Nématodes des ruminants domestiques.

\section{ETUDE DU POUVOIR ANTHELMINTHIQUE DU THIABENDAZOLE : RÉSULTATS AU LABORATOIRE}
A. - Témoins (Tableav No II)
B. - Action sưr les Trématodes

Quelle que soit la dose utilisée (de 100 à $400 \mathrm{mg} / \mathrm{kg}$ ), le médicament est dénué de toute activité à l'égard de Schistosoma bovis des veines hématiques et mésentériques.

\section{C. - Action sur les Cestodes (Tableau No HI)}

Le Thiabendazole est totalement inefficace sur les grands Cestodes de l'intestin du dromadaire, sauf à fortes doses où un certain nombre d'Avitellina sont chassés. Les résultats sont néanmoins irréguliers.

A plus faibles doses, l'expulsion est le fait de Cestodes en cours d'élimination naturelie. 


\section{MABLELO NOIII}

Cestodes

Nombre d'aninaur déparasités après traitement au Thíabendazole.

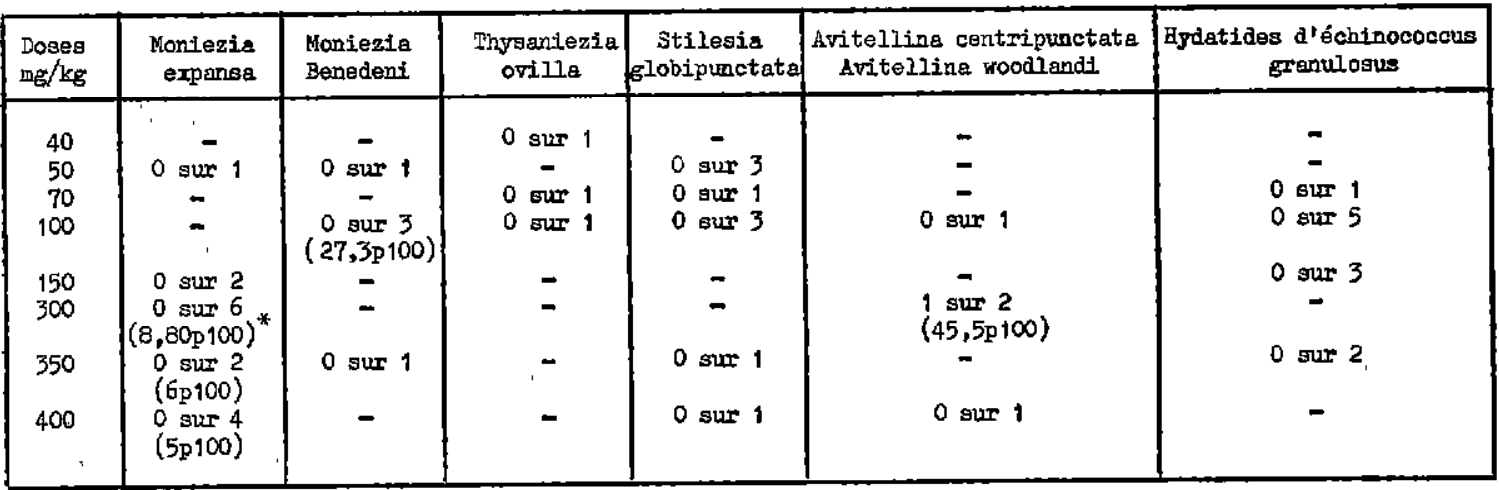

*'Quelques fragments de Cestodes sont expulsés aprés adminiatration du médicament.

Avec Moniezia expansa ef Avitellina centripunctota, le même phénomène a été observé chez le mouton traité au Thiabendazole.

\section{D. - Action sur les Nématodes}

10 Tableau No IV : examens coproscopiques. La lecture du Tableau No IX appelle les remarques suivantes :

- Le nombre d'cufs au gramme de matière fécale est élevé en juin-juillet (100 et $150 \mathrm{mg} / \mathrm{kg}$ ), bien qu'à l'autopsie, un grand nombre d'Haemoncus longistipes soient encore immatures.

- A partir de $100 \mathrm{mg} / \mathrm{kg}$. la baisse constatée le dernier jour n'est que faiblement imputable à la destruction d'Haemoncus longistipes. Plus vraisemblablement, elle est liée d'une part à une légère diminution du nombre d'cufs au gramme, comme chez les témoıns, d'autre part à la destruction quasi totale des Trichostrongylus et des Impalaia.

- Les Trichures semblent absents le dernier jour : it n'en est rien et, dans ce cas, la méthode des examens coprologiques conduit à des interprétations erronées.

$2^{\circ}$ Tableau No $V$ : action du Thiabendazole sur Strongyloides papillosus.

3o Tableau No VI : action du Thiabendazole sur Trichostrongylus vitrinus, Trichostrongylus probolurus ef impalaio nudicollis.

40 Tableau No VII : action du Thiabendazole sur Hoemoncus longistipes. 5o Tableau Na VIII : action du Thiabendazole sur Oesophagostomum adultes mûrs et immatures.

60 Action du Thiabendazole sur Buckleyuris globulosa.

70 Discussion.

a) Le Thiabendazole, quelle que soit la dose employée, est inactif sur Buckleyuris globulosa. Les Trichures - toujours nombreux - demeurent vivants dans la lumière caecale.

b) A partir de $70 \mathrm{mg} / \mathrm{kg}$. les' Strongyloides papillosus adultes disparaissent.

c) Vers $100 \mathrm{mg} / \mathrm{kg}$, les Heligmosomatidae (Impalaia nudicollis) et les Trichostrongylidae (T. vitrinus et $T$. probolurus) sont touchés et ne sont plus visibles lors du raclage de la muqueuse intestinale, ce que confirment d'ailleurs les examens coproscopiques.

d) Sur Qesophagostomum columbianum, les résultats sont irréguliers. Lorsqu'il s'agit de parasites adultes et mûrs, la dose de $100 \mathrm{mg} / \mathrm{kg}$ paraît suffisante : elle ne l'est plus quand on a affaire à des adultes immatures. Il faut alors des doses beaucoup plus importantes.

e) Sur Haemoncus longistipes, les résultats sont relativement bons, à partir de $70 \mathrm{mg} / \mathrm{kg}$. si les. Helminthes sont peu nombreux et sexuellement mûrs.

Par contre, dès lors que le parasitisme est massif (de 150 à 3.000 unités et plus) ef les Haemoncus encore immatures - comme ce fut le cas en juin-juillet 1965 - il importe de distribuer des doses très fortes de l'ordre de $300-350 \mathrm{mg} / \mathrm{kg}$. 
TABLEAU NOIV

Moyenne du nombre d'oeufo au bramne de matière fécale.

\begin{tabular}{|c|c|c|c|c|c|c|c|c|c|}
\hline \multirow{2}{*}{$\begin{array}{l}\text { Doses } \\
\mathrm{mg} / \mathrm{kg}\end{array}$} & \multicolumn{3}{|c|}{ Avant traitement } & \multicolumn{3}{|c|}{ Aprèg traitement } & \multicolumn{3}{|c|}{ Dertier jour } \\
\hline & Stz. & Tr. & Strl. & Str. & $\operatorname{Tr}$. & Strl. & Str. & Tr. & Strl. \\
\hline $40+$ & - & 20 & - & - & 51 & - & - & 0 & - \\
\hline $50+++$ & 25 & 6 & 6 & 5 & 25 & 18 & 0 & 210 & 0 \\
\hline $70++$ & 18 & - & - & - & - & - & - & - & - \\
\hline $100+111+1$ & 455 & 12 & 0 & 345 & 28 & - & 240 & 105 & - \\
\hline $150++++++$ & 1.355 & - & - & 1.235 & 4 & - & 145 & - & - \\
\hline $300+1+$ & 594 & - & - & 224 & - & - & 26 & 52 & - \\
\hline $350+++$ & 253 & - & - & 74 & - & 5 & - & - & - \\
\hline $400+x+4$ & 50 & $\infty$ & - & 177 & 3 & - & - & - & - \\
\hline $500+$ & 295 & - & - & 76 & 0 & 0 & - & - & - \\
\hline $1.000+$ & 262 & - & - & 23 & - & - & - & - & - \\
\hline & 211 & - & - & 165 & - & - & 157 & - & - \\
\hline
\end{tabular}

Str. = Haemoncus longistipes, Impalaia nudicollis.

Tr. = Trichostrongylus vitrinus, Prichostrongylus probolurus, Trichuris globulosa.

Strl.= Stronerloides pepillosus.

TABITAU NOV

Action du Thiabendazole sur strongyloides papillosus aduites

\begin{tabular}{|c|c|c|c|c|c|c|}
\hline $\begin{array}{l}\text { Doses } \\
\mathrm{mg} / \mathrm{kg}\end{array}$ & $\begin{array}{c}\text { Nombre } \\
\text { d'animaux } \\
\text { parasités }\end{array}$ & $\begin{array}{r}\text { Culture } \\
\text { ivombre total } \\
\text { Avent trai tement }\end{array}$ & $\begin{array}{l}\text { d'oeufs } \\
\text { de larves } I_{1}{ }^{*} \\
\text { |Après trai toment }\end{array}$ & $\begin{array}{l}\text { Autopsie } \\
\text { Hombre d'anımaux } \\
\text { encore parasités }\end{array}$ & Efficacité & Epoque des traitements \\
\hline 50 & 3 & 422 & 368 & 2 sur 3 & $12,3 p \cdot 100$ & Février 1965 \\
\hline 70 & 3 & 31 & 0 & 3 sur 3 & 100 & Février 1965 \\
\hline 100 & 5 & 32 & 0 & $5 \operatorname{sur} 5$ & 100 & Février et Juin 1965 \\
\hline 150 & 2 & 18 & 0 & $2 \operatorname{sus} 2$ & 100 & Juin 1965 \\
\hline 300 & 3 & 7 & 0 & $3 \sin 3$ & 100 & Novembre 1965 \\
\hline 350 & 3 & 64 & 0 & $3 \sin 3$ & 100 & $n$ \\
\hline 400 & 4 & 492 & 0 & $4 \operatorname{sur} 4$ & 100 & " \\
\hline 500 & 2 & 12 & 0 & 2 aur 2 & 100 & Juin 1965 \\
\hline
\end{tabular}

* Dars une goutte de suspension aqueuse provenant des boftes de Pétri ayant servi aux coprocultures.

TABLEAU NOVI

Action du Thiabendazole sux I'richostrol.cylus vitrinus, Trichostrongylus probalurus at Impalaia nudicollis

\begin{tabular}{|c|c|c|c|c|}
\hline $\begin{array}{l}\text { Doseg } \\
\mathrm{mg} / \mathrm{kg}\end{array}$ & $\begin{array}{l}\text { irombre } \\
\text { i'animawx } \\
\text { parasités }\end{array}$ & $\begin{array}{l}\text { Nombre d'animaux } \\
\text { complètement } \\
\text { déparasités }\end{array}$ & $\begin{array}{c}\text { Présence ou absence }(-) \\
\text { de Nématodes à } \\
\text { I'autopsie (moyenne) }\end{array}$ & Epoque des traitements \\
\hline 50 & 2 & 0 & 2 & Février 1965 \\
\hline 70 & 2 & 0 & 6 & $"$ \\
\hline 100 & 2 & 2 & - & Février et Jwin 1965 \\
\hline 150 & 3 & 3 & - & Juin 1965 \\
\hline 500 & 1 & 1 & - & Juin 1965 \\
\hline
\end{tabular}


THB:BLAU I:OVII

Action du Thiebend\&zole stur Haemoncus longistipes.

\begin{tabular}{|c|c|c|c|c|c|c|c|c|c|}
\hline Doseg $\mathrm{mg} / \mathrm{kg}$ & 40 & 50 & 70 & 100 & 150 & 300 & 350 & 400 & 500 \\
\hline Nombre de chameaur utilisés & 1 & 2 & 2 & 7 & 5 & 4 & 2 & 4 & 2 \\
\hline $\begin{array}{l}\text { Nombre de chameaux } \\
\text { totalement déparasités }\end{array}$ & 0 & 0 & 2 & 2 & 2 & 1 & 1 & 3 & 2 \\
\hline $\begin{array}{l}\text { ivombre total de } \\
\text { parasites expulsés }\end{array}$ & 0 & 0 & 0 & 0 & 0 & 6 & 0 & 0 & 0 \\
\hline $\begin{array}{l}\text { Nombre total de parasites } \\
\text { restant à } l^{\prime} \text { autopsie }\end{array}$ & 78 & 160 & 0 & 6.045 & 4740 & 190 & 1 & 4 & 0 \\
\hline $\begin{array}{l}\text { Nombre total de larvea } \mathrm{L}_{3} \\
\text { en coprocuiture } \\
\text { - avant traitement* } \\
\text { - après traitement }\end{array}$ & $\begin{array}{l}1 \\
1\end{array}$ & $\begin{array}{l}3 \\
3\end{array}$ & $\begin{array}{l}6 \\
0\end{array}$ & $\begin{array}{l}196 \\
105\end{array}$ & $\begin{array}{r}196 \\
18\end{array}$ & $\begin{array}{r}35 \\
5\end{array}$ & $\begin{array}{l}7 \\
0\end{array}$ & $\begin{array}{r}33 \\
0\end{array}$ & $\begin{array}{l}3 \\
0\end{array}$ \\
\hline $\begin{array}{l}\text { Efficacité } \\
\text { - chameaux faiblement } \\
\text { parasités } \\
\text { - chameaux fortement } \\
\text { parasités }\end{array}$ & $\begin{array}{c}\text { nuile } \\
-\end{array}$ & $\begin{array}{c}\text { nuzle } \\
-\end{array}$ & $\begin{array}{c}\text { bovine } \\
-\end{array}$ & $\begin{array}{c}- \\
\text { faible }\end{array}$ & faible & $\begin{array}{c}- \\
85 p 100\end{array}$ & $\begin{array}{c}- \\
90 p 100\end{array}$ & $\begin{array}{c}- \\
95 \mathrm{p} 100\end{array}$ & $\begin{array}{c}- \\
100 p 100\end{array}$ \\
\hline Epoque des traitements & $\begin{array}{l}\text { Févriex } \\
1965\end{array}$ & $\begin{array}{l}\text { Pévrler } \\
1965\end{array}$ & $\begin{array}{l}\text { Février } \\
1965\end{array}$ & $\begin{array}{l}\text { Février } \\
\text { Juin } 65\end{array}$ & $\begin{array}{l}\text { Juin } \\
1965\end{array}$ & $\begin{array}{l}\text { Novembre } \\
1965\end{array}$ & $\begin{array}{c}\text { Novembre } \\
1965\end{array}$ & $\begin{array}{c}\text { iNavembre } \\
1965\end{array}$ & $\begin{array}{l}\text { Juin } \\
1965\end{array}$ \\
\hline
\end{tabular}

* dans une goutte de susdension aqueuse provenant des boîtes de Pétri ayent servi aux coprocultures.

TABLEASU NOVIII

Action du Thiabendezole sur Desophagostomum columbianum mûrs et jomatures.

\begin{tabular}{|c|c|c|c|c|c|}
\hline $\begin{array}{l}\text { Doses } \\
\mathrm{mg} / \mathrm{kg}\end{array}$ & $\begin{array}{l}\text { Nombre } \\
\mathrm{d}^{2} \text { aninaaux } \\
\text { parasités }\end{array}$ & $\begin{array}{c}\text { Nombre } \\
\text { d'Oesophagostomes } \\
\text { expulsés }\end{array}$ & $\begin{array}{l}\text { Présence ou absence } \\
\text { de parasites à l'autopsie }\end{array}$ & $\begin{array}{l}\text { Nombre d'animaux } \\
\text { totalement déparesités }\end{array}$ & $\begin{array}{l}\text { Epoque des } \\
\text { traitements }\end{array}$ \\
\hline 50 & 3 & 0 & 143 & 0 sur 3 & Février 1965 \\
\hline 100 & 5 & 23 & 1 & 5 sur 5 & Février - \\
\hline 150 & 6 & 31 & 89 & $2 \operatorname{sur} 6$ & Juin 1965 \\
\hline 300 & 3 & 24 & 0 & 3 sur 3 & Novembre 1965 \\
\hline 350 & 2 & 4 & 0 & $2 \sin 2$ & Novembre 1965 \\
\hline 400 & 4 & 1 & 0 & 4 sur 4 & Novembre 1965 \\
\hline 500 & $1++$ & 0 & 0 & 1 sur 1 & Juin 1965 \\
\hline 1000 & $1++$ & 0 & 0 & 1 sur 1 & Juin 1965 \\
\hline
\end{tabular}

+ prégence d'un grand nombre d'Oesophagostomes adultes et inmatures sur coprocuiturea. 
TABLEAU NOIX

Action du Thuabendazole sur Buckleyuris globulosa.

\begin{tabular}{|c|c|c|c|c|c|}
\hline $\begin{array}{l}\text { Doses } \\
\mathrm{mg} / \mathrm{kg}\end{array}$ & $\begin{array}{c}\text { Nombre } \\
\text { d'animaux } \\
\text { parasités }\end{array}$ & $\begin{array}{l}\text { Nombre } \\
\text { de bucicleyuris } \\
\text { expulsés }\end{array}$ & $\begin{array}{l}\text { Présence ou àsence } \\
\text { de Nématodes à l'autopsie }\end{array}$ & $\begin{array}{l}\text { Nombre d'animaux } \\
\text { totalement déparasités }\end{array}$ & $\begin{array}{l}\text { Epoque des } \\
\text { traitements }\end{array}$ \\
\hline 40 & 1 & 0 & 32 & 0 sur 1 & Février 1965 \\
\hline 50 & 3 & 1 & 215 & 0 sur 3 & Fóvrier 1965 \\
\hline 70 & 3 & $t$ & 24 & $0 \operatorname{sur} 3$ & Février 1965 \\
\hline 100 & 6 & 1 & 524 & 0 sur 6 & Février-Jwin 1965 \\
\hline 150 & 5 & 0 & 101 & 0 sur 6 & Juin 1965 \\
\hline 300 & 4 & 2 & $\uparrow 03$ & 0 sus 4 & Novemise 1965 \\
\hline 350 & 3 & 0 & 23 & 0 sur 3 & Novembre 1965 \\
\hline 400 & 4 & 0 & 228 & 0 sur 4 & Novembre 1965 \\
\hline
\end{tabular}

Le pourcentage d'efficacité oscille alors autour de $85-90$ p. 100. II existe donc une différence considérable entre le pouvoir anthelminthique du Thiabendazole sur Haemoncus contortus du mouton et du bøuf qui ne résiste pas à des doses de $60-80 \mathrm{mg} / \mathrm{kg}$ et l'action du même médicament sur Haemoncus longistipes du chameau qui requiert des doses élevées : le résultat est certes intéressant, mais il n'est jamais entièrement positif, en ce sens que l'élimination compiète des Strongles de la caillette n'est pas assurée. L'origine exacte de cette différence reste inconnue et, pour l'instant, aucune explication satisfaisante ne peut être donnée.

f) En définitive, deux dosages sont susceptibles d'être préconisés :

— de 100 à $150 \mathrm{mg} / \mathrm{kg}(*)$. Cette dose est recommandée si l'on soupçonne l'existence de IStrongyloides papillosus, Trichostrongylus vitrinus, Trichostrongylus probolurus ef Impalaia nudicollis. L'association parasitaire est en partie désorganisée et un ceríain nombre d'espèces dangereuses supprimées. L'état sanitaire de l'animal s'améliore rapidement et son équilibre biologique se rétablit peu à peu, ainsi qu'il sera dit plus loin.

C'est ce qui a été constaté à la fin de 1965 dans la région d'Ati où des chameaux très anémiés ef suspects de Trypanosomiase, ont été, faute d'un Trypanocide, traités systématiquement au Thiabendazole à faıble dose avec de remarquables effets.

- $300 \mathrm{mg} / \mathrm{kg}\left({ }^{*}\right)$ qui représente la véritable

(*) de Thiabendazole pur. dose polyvalente capable d'agir à la fois sur les Strongyloides, les Trichostrongylus, les Impalaia, 85 p. 100 des Haemoncus longistipes et la quasitotalité des Oesophagostomum. Au Tchad où les Nématodes de la cajllette et de l'intestin sont presque constamment associés, elle doit être retenue de préférence à la précédente et sera utilisée de mai à novembre, mois où le parasitisme gastro-intestinal est le plus abondant ef le plus nocif.

\section{E. - Action sur Globidium comeli (Tableau No $X$ ).}

II ne peut être fait usage du Thiabendazole dans le traitement de la globidiose cameline, le nombre d'oocystes ne diminuant pas sensiblement en fin de traitement.

\section{TABLEAO NOX}

Action du Thilebendazole sur Globidium cemeli.

Woyenne du nombro d'oocystes au gramme de matière fécele.

\begin{tabular}{|l|c|c|c|}
\hline $\begin{array}{l}\text { Doses } \\
\text { mg } / \mathrm{kg}\end{array}$ & $\begin{array}{c}\text { Avant } \\
\text { traitement }\end{array}$ & $\begin{array}{c}\text { Pendent } \\
\text { traitement }\end{array}$ & $\begin{array}{l}\text { Dernier } \\
\text { jour }\end{array}$ \\
\hline $50+$ & 160 & 245 & 105 \\
$100+++$ & 153 & 103 & 387 \\
$150++$ & 0 & 72 & 15 \\
$300+++$ & 17 & 12 & 70 \\
$400+$ & 26 & 0 & 20 \\
\hline
\end{tabular}

$t=$ une crolx représente un animal. 


\section{CONSÉQUENCES DU TRAITEMENT SUR LA SANTÉ DE L'ANIMAL}

\section{Conséquences visibles.}

Dans les conditions du laborataire, le médicament est assez bien supporté ef ne provoque pas de perturbations notables dans le comportement de l'animal. L'appétit reprend ou croît rapidement. Le foin a tendance à être consommé en plus grande quantité par les animaux traités que par les témoins.

2. Numérations globulaires. Formules leucocytaires.

Les variations sont plus ou moins marquées selon la saison, la dose, l'origine des animaux et l'intensité du parasitisme.

- Sur des chameaux peu anémiés et traités à $70-100 \mathrm{mg} / \mathrm{kg}$, on observe une légère diminution du nombre d'hématies et un accroissement 'des leucocytes. Les Eosinophiles baissent légèrement.

- Sur des chameaux affaiblis (Kanem, novembre 1965), fortement parasités ef ayant reçu $300 \mathrm{mg} / \mathrm{kg}$, les résultats sont bien meilleurs. Le taux d'hématies remonte nettement $(+10 \mathrm{p}$. 100). Pour les éosinophiles, la diminution est supérieure à 50 p. 100.

\section{Protéines du sang.}

Elles ont fait l'objet d'une étude approfondie qui bientôt sera publiée (QUEVAL, GRABER et BRUNET). Le Tableau No XI résume globalement les constatations faites.

Chez le chameau en bon état, les constantes protéiniques sont approximativement :

Protéines totales : 83,9 .

Albumines : 34,8 .

Globulines: 49,1 .

A 150 et à $300 \mathrm{mg} / \mathrm{kg}$, les Protéines totales augmentent d'un peu plus de 15 p. 100 au bénéfice des Albumines ( $+9,6$ p. 100 et $+21,5$ p. 100) ef des globulines, ce qui correspond dons le premier cas à la disparition de tous les Trichostrongylidae, Heligmosomatidae et Rhabditidae, et, dans le second, de tous les Nématodes associés à l'exception d'un petit nombre d'Haemoncus et de Buckleyuris globulosa.

A $400 \mathrm{mg} / \mathrm{kg}$, les conclusions sont moins nettes, car il s'agissait au départ d'animaux moyennement parasités, avec un taux de Protéines totales proche de la normale. Le rapport Albumine/Globuline passe alors de 0.46 à 0,52 .

\section{Prises de poids.}

Deux essais ont été réalisés :

- d̀ $300 \mathrm{mg} / \mathrm{kg}$.

- d̀ $400 \mathrm{mg} / \mathrm{kg}$.

le premier en novembre 1965 sur ides dromadaires originaires du Kanem et, le second, en décembre sur des chameaux venus du Batha. Dans les deux cas, les animaux (quatre dans chaque série) étaient atteints d'un polyparasitisme à base de Nématodes et de Cestodes.

La durée de l'expérience a été de 18 jours.

Les animaux ont été nourris avec des foins composés de Brachiaria, d'Echinochloo et de Panicum. Les analyses (*) ont montré que les foins récoltés en octobre assuraient une ration d'entretien suffisante, ce qui n'était plus le cas en décembre avec des foins de même nature, mais ramassés plus tard dans la saison. C'est ce que démontrent les deux tableaux suivants : (Tableaux XII ef XIII).

Chez les chameaux, les résultats sont les suivants: (TableaU XIV)

L'expérience prouve qu'après traitement au Thiabendazole administré à forte dose, si les conditions alimentaires sont satisfaisantes, la reprise de poids est rapide et importante. Dans le cas contraire, le traitement ne donne que de médiocres résultats.

Il importe donc, après le déparasitage, de placer les chameaux au repos sur dés pâturages herbacés ou arbustifs abondants et fournis. Ce détail a une importance considérable.

A $300 \mathrm{mg} / \mathrm{kg}$, le gaın de poids est à mettre en parallèle avec :

- l'augmentation du nombre d'hématies $(+10$ p. 100) ;

- l'augmentation des protéines totales $(+15$ p. 100) ;

- In diminution du nombre d'Haemoncus longistipes (-85 p. 100) et la disparition des Rhabditidae, des Trichostrongylidae et des Heligmosomatidae de l'intestin.

(*) Effectuées à l'I. E. M. V. T. à Paris. 


\section{TABLFALU N}

Protéines du sang avant et après traitement au Triobendazole.

\begin{tabular}{|c|c|c|c|c|c|c|c|c|c|}
\hline \multirow{2}{*}{$\begin{array}{l}\text { Doseg } \\
\mathrm{mg}_{\mathrm{B}} / \mathrm{kg}_{\mathrm{g}}\end{array}$} & \multicolumn{3}{|c|}{ Protéines totales } & \multicolumn{2}{|c|}{ Alburines } & \multicolumn{2}{|c|}{ Globulines } & \multirow{2}{*}{$\begin{array}{l}\text { Oripine } \\
\text { des } \\
\text { enimeux }\end{array}$} & \multirow{2}{*}{$\begin{array}{l}\text { Epouve des } \\
\text { traitements }\end{array}$} \\
\hline & \begin{tabular}{|c|} 
Avant \\
treattement
\end{tabular} & $\begin{array}{c}\text { Après } \\
\text { treiter. }\end{array}$ & $\begin{array}{l}\text { Pourcentage } \\
\text { Augmentation }\end{array}$ & $\begin{array}{c}\text { Avant } \\
\text { trajter. }\end{array}$ & $\begin{array}{l}\text { Après } \\
\text { traitem. }\end{array}$ & $\begin{array}{c}\text { Avant } \\
\text { treil tea. }\end{array}$ & $\begin{array}{c}\text { Après } \\
\text { traitem. }\end{array}$ & & \\
\hline 70 & 67 & 67 & $=$ & 20,2 & 21,5 & 46,6 & 45,5 & Kanem & Février 1965 \\
\hline 100 & 67,1 & 70,3 & $+4,7 p 100$ & 22,2 & 19,1 & 45,9 & 51,1 & Kanem & Fétrier 1965 \\
\hline 150 & 71,75 & 83,1 & $+15,8 \mathrm{p} 100$ & 24,6 & 29,9 & 47,1 & 53,2 & Bathe & Juin 1965 \\
\hline 300 & 73,8 & 85,4 & $+15.7 p 100$ & 26,1 & 28,6 & 47,7 & 56,8 & Kanem & Detobre 1965 \\
\hline 400 & 81,5 & 81,7 & $+0,2 p 100$ & 25,9 & 28,1 & 55,6 & 53,6 & Bathe & Novembre 1965 \\
\hline
\end{tabular}

TABIEAAU NOOZI

Anelyses de fourrage - Pourcentage de la matière brute.

\begin{tabular}{|c|c|c|}
\hline & Octobre 1965 & Décombre 1965 \\
\hline $\begin{array}{l}\text { Hunidité } \\
\text { Matière sèche } \\
\text { Matières protéiques brutes } \\
\text { Cellulose } \\
\text { Matières grasses (ertrait éthéré) } \\
\text { Matières minérales totales } \\
\text { Ertractif non azoté } \\
\text { Insoluble chlorhydrique } \\
\text { Calcium } \\
\text { Phosphore } \\
\text { Magnésium } \\
\text { Potassium } \\
\text { Sodium. }\end{array}$ & $\begin{array}{r}40,10 \\
59,90 \\
1,39 \\
24,15 \\
0,47 \\
7,94 \\
25,95 \\
5,34 \\
0,182 \\
0,053 \\
- \\
1,01 \\
-\end{array}$ & $\begin{array}{c}5,90 \\
94,10 \\
5,34 \\
36,50 \\
0,82 \\
12,75 \\
38,69 \\
6,79 \\
0,325 \\
0,351 \\
- \\
2,58 \\
-\end{array}$ \\
\hline
\end{tabular}

TABIEAUU WOXIIT

Analyses de fourrage - Pourcentage de la matière sèche

\begin{tabular}{|c|c|c|}
\hline & Ostobre 1965 & Décembre 1965 \\
\hline $\begin{array}{l}\text { Humidité } \\
\text { Matière sèche } \\
\text { Matières protéiques brutes } \\
\text { Cellulose } \\
\text { Matières grasses (extrait éthéré) } \\
\text { Matières minérales totales } \\
\text { Extractif non azoté } \\
\text { Insoluble chlorhydrique } \\
\text { Caj cium } \\
\text { Phosphore } \\
\text { Megnésium } \\
\text { Potassium } \\
\text { Sodium }\end{array}$ & $\begin{array}{c}- \\
100,00 \\
2,32 \\
40,35 \\
0,79 \\
13,26 \\
43,28 \\
8,92 \\
0,304 \\
0,089 \\
- \\
1,68 \\
-\end{array}$ & $\begin{array}{l}-\overline{0}, 00 \\
5,67 \\
38,80 \\
0,87 \\
13,55 \\
41,11 \\
7,22 \\
0,345 \\
0,373 \\
= \\
2,74 \\
=\end{array}$ \\
\hline
\end{tabular}

TABLARUU NOXIV

Prises de poida

\begin{tabular}{|c|c|c|c|}
\hline Doses en mg $/ \mathrm{kg}$ & 300 & 400 & Témoins \\
\hline $\begin{array}{l}\text { Poids total des arimaur avant traitement } \\
\text { (en kilograrmes) }\end{array}$ & $1.209,8$ & $1.340,7$ & 668,3 \\
\hline $\begin{array}{l}\text { Poids totel des animaux après traitement } \\
\text { (en kilogrammes) }\end{array}$ & $1.345,6$ & $1.368,2$ & 662,9 \\
\hline Norbse d'arimaus & 4 & 4 & 2 \\
\hline Pourcentage d'augmentation & $+11 p \cdot 100$ & +2 p. 100 & $-0,9 \mathrm{p} \cdot 100$ \\
\hline
\end{tabular}




\section{ACTIVITÉ DU MÉDICAMENT}

L'évacuation des parasites, lorsqu'elle a lieu, débute 48 heures après l'administration de l'anthelminthique. Elle dure assez longtemps et ne paraît terminée qu'au bout de cinq jours. Seuls, les Oesophagostomes sont éliminés intacts. Peu d'Haemoncus sont visibles dans les crottes : ils sont pour la plupart en partie digérés.

A $300 \mathrm{mg} / \mathrm{kg}$, au bout d'une quinzaine de jours, les œufs, lorsque survivent des parasites môrs (Hoemoncus), peuvent donner naissance à des larves $L_{3}$ bien vivantes, mais en très petit nombre.

\section{TOXICITÉ}

Des doses progressivement croissantes ont été expérimentées (Tableau XV).

Le Thiabendazole est, dans l'ensemble, assez bien toléré, même à des doses de $1.000 \mathrm{mg} / \mathrm{kg}$. Cependant, vers $350-400 \mathrm{mg} / \mathrm{kg}$, des accidents mortels sont susceptibles de se produire. Ils sont le fait d'animaux très maigres, très anémiés, souvent atteints de Trypanosomiase, d'Echinococcose ef d'abcès multiples.

Il importe donc dans un troupeau, avant le traitement, de rechercher systématiquement tous les porteurs de Trypanosomes ou les animaux trop malades (abcès; broncho-pneumonies) qui seront séparés et soignés. Ils ne seront dépara- sités qu'un peu plus tard au moyen d'une dose de Thiabendazole réduite de moitié $(150 \mathrm{mg} / \mathrm{kg}$ ). La dose normale $(300 \mathrm{mg} / \mathrm{kg}$ ) ne sera distribuée que quand l'animal sera capable de la supporter.

Les signes de l'intoxication par le Thiabendazole sont peu manifestes. Les conséquences à long terme se traduisent essentiellement par une baisse de poids régulière qui pour le chameau No $6(350 \mathrm{mg} / \mathrm{kg})$ se présentait ainsi :

30-11-1965 (traltement) : $316,5 \mathrm{~kg}$.

4-12-1965: 301,5 kg.

$11-12-1965$ (mort) : $281,8 \mathrm{~kg}$.

Le même phénomène a été observé chez des équidés en mauvais état ayant reçu de fortes doses de Thiabendazole.

\section{ESSAIS SUR LE TERRAIN}

1. Choix d'une méthode barymétrique permettant d'apprécier le poids d'un dromadaire quand il n'existe pas d'instrument de pesée dans une réglon donnée.

Avant d'étudier l'action du Thiabendazole sur le terrain, il importait de choisir une méthode barymétrique permettant, en l'absence de tout instrument, de calculer le poids des animaux devant être traités.

C'est une variante de la méthode de BOUÉ (1949) qui a été adoptée. Elle consiste, pour

TABLEAU NOXY

Toxicite du Thibendazole pour le dromadaire

\begin{tabular}{|c|c|c|c|c|}
\hline $\begin{array}{l}\text { Doses } \\
\mathrm{mg} / \mathrm{kg}\end{array}$ & $\begin{array}{c}\text { Nombre de } \\
\text { chameaur utilisés }\end{array}$ & Mortalité & $\begin{array}{l}\text { Epoque des } \\
\text { traitements }\end{array}$ & $\begin{array}{l}\text { Etat général } \\
\text { des anjmaux }\end{array}$ \\
\hline 40 & 1 & 0 sur 1 & Février & Moyen \\
\hline 50 & 3 & 0 sur 3 & $"$ & $u$ \\
\hline 70 & 3 & $0 \sin 5$ & $n$ & $"$ \\
\hline 100 & 7 & $a \operatorname{sur} 7$ & Février-Juin & Meuvais \\
\hline 150 & 5 & 0 sur 5 & Juin & " \\
\hline 300 & 4 & 0 gur 4 & Octobre & Moyen \\
\hline 350 & 3 & 1 aur 3 & Novembre & Mauvais \\
\hline 400 & 4 & 0 sur 4 & Novembre & Moyen \\
\hline 500 & 2 & $0 \sin 2$ & Jutn & Mauvais \\
\hline 1.000 & 1 & 0 sur 1 & $"$ & $n$ \\
\hline
\end{tabular}


TABLRAU NNOXVI

Evaluation de la relation moyenne

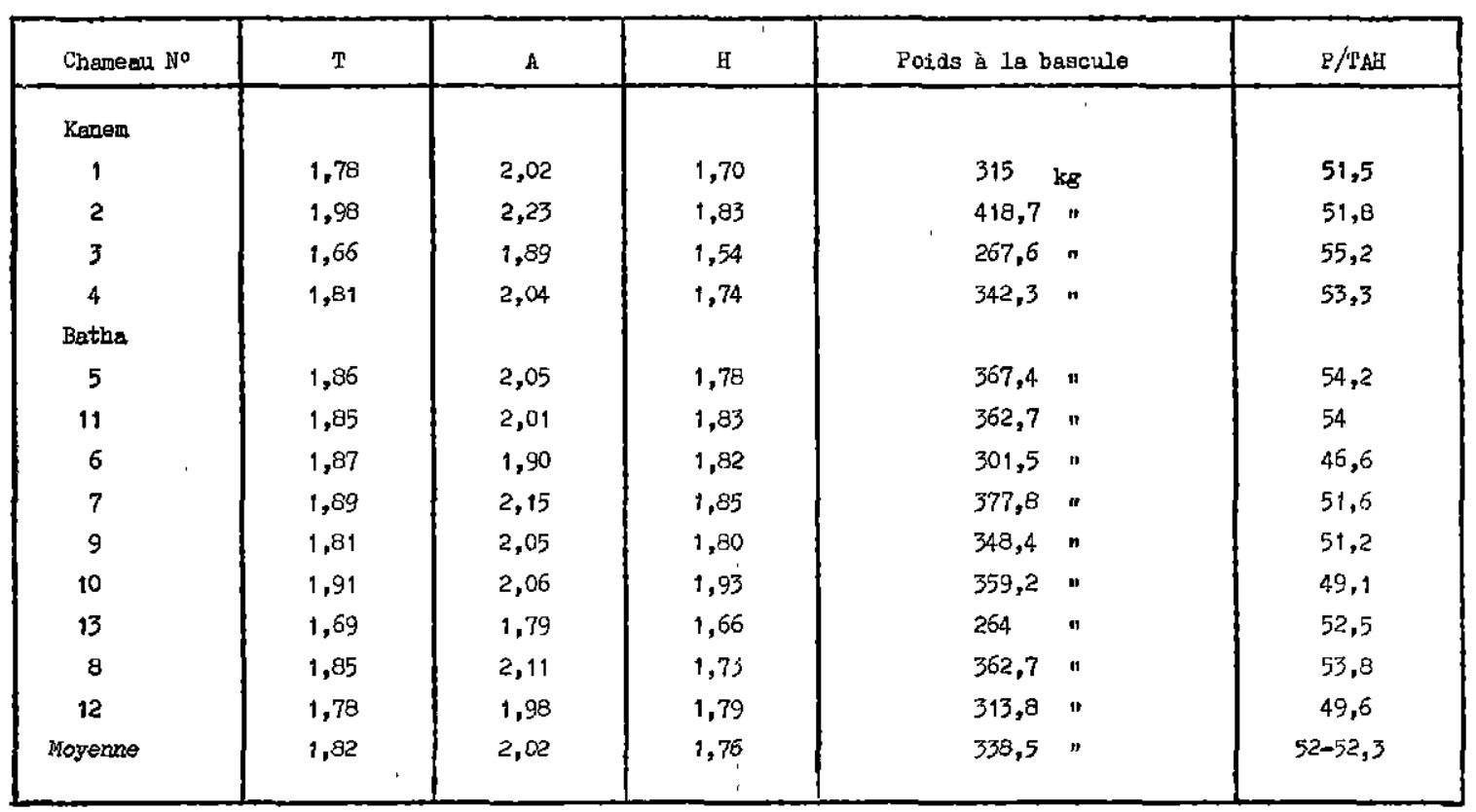

$\mathrm{L}_{\text {a }}$ chiffre 52 a finalenent été retenu pour la relation moyenne P/TAH

TABLEAU NaKVII

Foids de I'enimal et approximation moyenne

\begin{tabular}{|c|c|c|c|c|}
\hline Chsmeau No & TAH & $\begin{array}{c}\text { TAH } \times 52 \\
\text { (kilogtammes) }\end{array}$ & $\begin{array}{l}\text { Poids à la bascule } \\
\text { (kilogrenmes) }\end{array}$ & $\begin{array}{l}\text { Approximation } \\
\text { (kilogranmes) }\end{array}$ \\
\hline 12 & 6,308 & 328 & 313,8 & $+14,2$ \\
\hline 13 & 5,021 & 261 & 264 & -3 \\
\hline 8 & 6,730 & 350 & 362 & -12 \\
\hline 10 & 7,2 & 374,4 & 359,2 & $+15,2$ \\
\hline 9 & 6,287 & 326,9 & 348,8 & $-21,9$ \\
\hline 7 & 6,872 & 357,3 & 377,8 & -20 \\
\hline 11 & 6,804 & 353.8 & 362,7 & $-8,9$ \\
\hline 5 & 6,787 & 353 & 367 & -14 \\
\hline$\eta$ & 6,112 & 317,8 & 315 & $+2,8$ \\
\hline 2 & 8,080 & 420,1 & 418,7 & $+1,4$ \\
\hline 3 & 4,862 & 252,8 & 267,6 & $-14,8$ \\
\hline 4 & 6,424 & 334 & 342,3 & $-8,3$ \\
\hline
\end{tabular}


obtenir le poids net d'un chameau du Sud Algérien, d̀ utiliser la formule suivante :

Poids nef $=$ Périmètre thoracique $\left(T^{*}\right) \times$ Périmètre abdominal $\left(A^{*}\right) \times$ Hauteur $\left(H^{*}\right) \times 53$.

- Le périmètre thoracique $(T)$ est pris sur l'animal debout, derrière la callosité sternale au moyen d'un ruban étalonné que l'on passe autour du corps.

- Le périmètre abdominal (A) à l'endroit le plus haut de la bosse, en son milieu et en fin d'expiration.

- La hauteur est mesurée au moyen d'une toise placée d̀ l'aplomb normal du membre antérieur, à la hauteur de la pointe du coude au milieu de l'avant-bras.

La formule ainsi définie, appliquée aux dromadaires du Tchad à leur arrivée au Laboratoire, révèle, par comparaison avec le poids réel à la bascule, des différences considérables, de l'ordre de 10 à 20 p. 100.

Il a donc fallu évaluer aussi exactement que possible le coefficient applicable aux dromadaires des zones sahélıennes du Tchad, le chiffre 53 n'étant valable apparemment que pour les Méharis du Sud-Algérien.

Pour ce faire, 13 chameaux originaires du Kanem et du Batha ont été utilisés et deux opérations effectuées :

- Evaluation de la relation moyenne (P/TAH) valable pour les chameaux de ces régions.

- En fonction du chiffre ainsi obtenu, appréciation du poids ef recherche de l'approximation moyenne.

Les animaux ont été maintenus dans des conditions semblables durant toute l'expérience. Ils ont été placés dans des étables, nourris avec de fortes rations de foin et abondamment abreuvés. Les mesures ont été faites le matin à 8 heures.

\section{A. - Evaluation de la relation moyenne (P/TAH) (Tableau XVI)}

Le chiffre 52 a finalement été retenu pour la relation moyenne $\mathrm{P} / \mathrm{TAH}$.

$$
\left(^{*}\right)=\text { en mètre. }
$$

\section{B. - Poids de l'animal et opproximation moyenne} (Tableau XVII)

La relation moyenne $\mathrm{P}=52 \mathrm{TAH}$ est bonne, car l'approximation ne dépasse pas $25 \mathrm{~kg}$ en plus ou en moins.

\section{Résultats sur le terrain}

Ils ont eu lieu en décembre 1965 à Abougoudam sur le peloton nomade du Ouaddaï composé de chameaux de sellefatigués et, dans l'ensemble, en assez mauvais état. Plus de la moitié d'entre eux hébergeaient en grand nombre Haemoncus longistipes, des Trichostrongylus, des impoloio, des Buckleyuris, ainsi que des filaires ef des Trypanosomes.

Par ailleurs, vu les faibles précipitations tombées au Ouaddai durant l'hivernage 1965, le pâturage était, en décembre, réduit et de qualité médiocre. Le poids des chameaux a été apprécié selon la formule précédente $P=52$ TAH et chacun a reçu $500 \mathrm{mg} / \mathrm{kg}$ environ de Thiabendazole commercial à 75 p. 100 . Sur 27 chameaux traités, 11 d'entre eux' ont manifesté une violente diarrhée $\left(^{*}\right)$ dans les jours qui ont suivi le traitement. La diarrhée rétrocède par la suite.

Cependant, deux animaux sont morts une douzaine de jours après l'administration du Thiabendazole. Il s'agissait de chameaux âgés, très maigres, usés, fortement parasités et porteurs de Trypanosomes.

Il a été alors décidé de scinder le troupeau en deux : les animaux les plus malades sont restés à la station (8), les autres ont été conduits plus au sud sur des pâturages arbustifs neufs et abondants. (Tableaux XVIII, XIX et XX).

L'observation a duré un mois et demi.

Il apparaît donc que, dans un effectif camelin médiocre après traitement au Thıabendazole à $500 \mathrm{mg} / \mathrm{kg}$ :

- Les animaux afteints d'autres affections (Trypanosomiase: abcès multiples; bronchopneumonies, etc...) tolèrent assez mal le médicament. Dans ce cas - comme il a été dił plus

$\left.{ }^{*}\right)$ Les chameliers ne craignent pas les conséquences néfastes de la diarrhée, car, d'après eux, elle signe un médicament de grande valeur. 
a) - 1er loi : Chameaux malades laissés à Abaugoudan.

TABLEAD NOTVIII

\begin{tabular}{|c|c|c|c|c|}
\hline Chameau No & $10-12-1965$ & $22-12-1965$ & $24-1-1966$ & Observations \\
\hline 1 & 525 & 535 & 546 & Filaires \\
2 & 438 & 443 & 419 & 538 \\
4 & 561 & & 492 & Trypanosomes \\
6 & 502 & 500 & 380 & 1 \\
7 & 412 & 406 & 337 & 1 \\
13 & 349 & 343 & 511 & 1 \\
22 & 555 & 538 & 341 & Trypanosomes \\
\hline
\end{tabular}

b) $-2^{e}$ lot : Chameaux remis sur un pâturage neuf.

TABLLEAU NOXIX

\begin{tabular}{|c|c|c|c|}
\hline Chameau No & $10-12-1965$ & $27-12-1965$ & $24-1-1966$ \\
\hline 5 & 415 & 438 & 438 \\
\hline a & 332 & 362 & 367 \\
\hline 10 & 440 & 455 & 442 \\
\hline 11 & 568 & 560 & 621 \\
\hline 12 & 465 & 437 & 467 \\
\hline 15 & 452 & 445 & 487 \\
\hline 16 & 417 & 415 & 442 \\
\hline 17 & 484 & 462 & 430 \\
\hline 19 & 494 & 472 & 493 \\
\hline 20 & 402 & 422 & 432 \\
\hline 23 & 518 & 539 & 577 \\
\hline 25 & 444 & 424 & 445 \\
\hline 26 & 331 & 322 & 343 \\
\hline 27 & 372 & 348 & 353 \\
\hline 28 & 324 . & 346 & 346 \\
\hline Poids moyen & 430 & 432,4 & 459,7 \\
\hline Gain & - & $+0,005 p 100$ & $+6,9 p 100$ \\
\hline
\end{tabular}

c) - Témoins: Chameaux remıs sur un pâturage neuf et riche.

MABIEAD NOXK

\begin{tabular}{|c|c|c|c|}
\hline Chamesu , No & $10-12-1965$ & $27-12-1965$ & $24-1-1966$ \\
\hline 29 & 335 & 350 & 351 \\
$1 ., 30$ & 449 & 433 & 437 \\
Poids mojyen & 392 & 391,5 & 394 \\
Augmentation & - & $-0,2 \mathrm{p} 100$ & $+0,005 p 100$ \\
\hline
\end{tabular}

* Les poids sont donnés en kilogrammes. 
haut - on a intérêt à éliminer la Trypanosomiase notamment et à ne distribuer qu'une dose minimum de Thiabendazole (150 $\mathrm{mg} / \mathrm{kg}$ par exemple) qui améliore l'état général du malade en supprimant les petits Nématodes du tube digestif. La dose complète destinée à tuer les Haemoncus ne sera administrée que lorsque le chameau sera capable de la supporter.

- Après traitement, les animaux doivent être placés au repos complet sur des pâturages convenables herbacés ef surtout arbustifs. Dans le cas de cette expérience. l'augmentation de poids a été de 6,9 p. 100 , celui des témoins placés dans les mêmes conditions ne variant pratiquement pas. Il est probable que, sur des dromadaires d'élevage, les résultats eussent été meilleurs encore, les éleveurs sachant choisir les parcours les plus riches. If est bon de noter que les gardiens du troupeau méhariste, outre l'accroissement de poids, ont constaté une régression importante et quasi générale de la gale qui touchait un assez grand nombre de chameaux. II faut voir là surtout une amélioration de l'état général.

\section{CONCLUSIONS}

Lors d'essais effectués sur 85 dromadaires adultes originaires du Kanem et du Batha (République du Tchad), il a été constaté que :

10 Le Thiabendazole, quelle que soit la dose utilisée, est complètement inactif sur Schistosoma bovis des veines mésentériques, Thysaniezia ovilla ef Stilesia globipunctoto de l'intestin, Echinococcus polymorphus du poumon et du foie, Globidium cameli de l'intestin, ef Buckleyuris globulasa du cæcum. L'action est faible et inconstanie sur Moniezia expanso, Moniezia benedeni, Avitellina centripunctata et Avitellina woodlandi de l'intestin. La plupart des scolex subsistent, même à fortes doses.

$2^{\circ}$ Les petits Nématodes intestinaux appartenant aux familles des Rhabditidae (Strongyloides papillosus), des Trichostrongylidae (Rrichostrongylus vitrunus ef Trichostrongylus probolurus ef des Heligmosomatidae (Impalaia nudicollis) sont tous détruits à partir de $100 \mathrm{mg} / \mathrm{kg}$.

3o Sur Oesophagostomum columbianum, les résultats sont irréguliers. Lorsqu'il s'agit de para- sites adultes et mûrs, la dose de $100 \mathrm{mg} / \mathrm{kg}$ paraît suffisante; elle ne l'est plus quand on a affaire à des adultes immatures. If faut alors des doses beaucoup plus importantes.

40 Sur Hoemoncus longistipes de la caillette, les résultats sont relativement bons à partir de $70 \mathrm{mg} / \mathrm{kg}$, si les Helminthes sont peu nombreux et sexuellement mûrs.

Par contre, dès que le parasitisme est massif (de 150 à 3.000 unités et plus) et les Haemoncus encore immatures, il importe de distribuer des doses fortes de l'ordre d'au moins $300 \mathrm{mg} / \mathrm{kg}$. Le pourcentage d'efficacité oscille alors autour de 85 p. 100.

$5^{0}$ En définitive, deux dosages sont susceptibles d'être préconisés :

- de 100 d̀ $150 \mathrm{mg} / \mathrm{kg}$ (produit pur). Cette dose est recommanidée si l'on soupçonne l'existence de Strongyloides papillosus, T. vitrinus, $T$. probolurus ef $I$. nudicollis. L'association parasitaire est en partie désorganisée et un certain nombre d'espèces dangereuses supprimées. L'état sanitaire de l'animal s'améliore rapidement ef son équilibre biologique se rétablit peu à peu :

- $300 \mathrm{mg} / \mathrm{kg}$ qui représente, pour le parasitologue, 'la véritable dose polyvalente capable d'agir à la fois sur les Strongyloides, les Trichostrongylus, les Impalaio, 85 p. 100 des Haemoncus longistipes ef la quasi-totalitédes Oesophogostomum: Au Tchad où les Nématodes de la caillette et de l'intestin sont presque constamment associés, elle doit être retenue de préférence et sera utilisée de mai à novembre, mois où le parasitisme gastro-intestinal du dromadaire est le plus abondant et le plus nocif.

$6^{\circ}$ Le Thiabendazole semble assez peu toxique, sauf pour les animaux très bas d'état, très anémiés, très parasités ou atteints d'autres affections (Trypanosomiase ; abcès multiples; pneumonies...). II faut alors traiter l'affection principale et ne traiter qu'avec une dose d'anthelminthique réduite de moitié, quitte à recommencer par la suite à la dose normale.

$7^{\circ}$ Le Thiabendazole, après destruction de la plus grande partie des Nématodes du tractus digestif du chameau, n'a d'heureuses répercussions sur l'état de santé de l'animal que dans 
la mesure où celui-ci est placé au repos sur un pâturage convenable. Les augmentations de poids sont alors' Importantes (de 6,8 à 11 p. 100 selon le cas). Elles s'accompagnent d'une remontée parallèle des Protéines du sang dont le taux tend alors vers la normale.

Le Thiabendazole est donc un anthelminthique parfaitement utilisable en Elevage camelin, à condition d'observer certaines règles et de prendre quelques précoutions.

\section{REMERCIEMENTS}

L'auteur tient à remercier vivement $M$. le Dr vétérinaire CAST qui a supervisé une partie des essais sur le terrain, $M$. le $D r$ vétérinaire RIVIERE qui a bien voulu procéder à l'analyse des fourrages expédiés de Fort-Lamy, Mme BRUNET et $M$. QUEVAL qui se sont particulièrement attachés à l'étude des formules sanguines et des Protéines du sang.

\section{SUMMARY}

Study of the parasiticide action of Thiabendazol on some helminths of domestic animals under some African conditions. II. Dromedary

From experimentation made in Chad in 85 Dromedaries from Kanem and Batha the author came to the conclusion that Thiabendazol is inactive on many Anoplocephalidae of the infestine, on Shistosoma bovis, on the Hydatid of Echinococcus gronulosus, on Buckleyuris globuloso and on Globidium comeli.

Two dosages can be recommended against the most common Nematodede of the stomach and the intestine :

- 100 to $150 \mathrm{mg} / \mathrm{kg}$ (of pure drug) : this dose is able to kill as a whole an association of Strongylaides papillosus, Trichostrongylus vitrinus, Trichostrongyius probolurus and Impolaio nudicallis Nematodeae, which are all particularly dangerous for the camel.

- $300 \mathrm{mg} / \mathrm{kg}$; this dose is for the parasitologist the most polyvalent, since it is active both on the above Helminths, on Haemoncus longistipes of the rennet (up to 85 p. 100) and on Oesophagostomum columbionum of the large intestine. In Chad this dose has to be preferred and used from may to november, when the parasitists of the stomach and the infestine of the dromedary are most numerous.

The Thiabendazole is not very toxicous for the camel, except if this animal is chronically infected by some other conditions (Trypanosomiasis, multiple abcesses, pneumonia, etc...). In that case the dose to be given must be reduced by half, but may be given again later.

After the treatment, the general state of health improves quickly, if the animal is fed on a suitable grazing, of a rather shrubby type. The gain of weight ranges from 6,8 p. 100 to 11 p. 100 .

This anthelminthic drung should be used in camel only if some precautions have been taken. 


\section{RESUMEN}

Estudio de la acción antiparasitaria del Thiabendazole en varios helmintos de los animales domésticos en ciertas condiciones africanas. II. Dromedario

A partir de ensayos efectuados en Chad en 85 dromedarios de Kanem y de Batha, el autor concluye que el Thiabendazale es inactivo en muchos Anoplocefolidae del intestino, en Shistosoma bovis, en los hidatides de Echinacoccus granulosus, en Buckleyuris globulosa y en Globidium cameli.

Pueden preconizar dos dosis contra los nemátodos gastro-intestinales más frecuentes :

- 100 a $150 \mathrm{mg} / \mathrm{kg}$ (producto puro). Esta dosis puede destruir totalmente una asociación de Strangyloides popillosus, Trichostrongylus vitrinus, Trichosirongylus probolurus e Impalaia nudicollis, nemátodos particularmente peligrosos para el camello.

- $300 \mathrm{mg} / \mathrm{kg}$. Esta dosis es la más polivalente para el parasitologo porque puede actuar en los dichos helmîntos como en Haemoncus longistipes del cuajar (a 85 por 100) y en Oesofagostomum columbianum del intestino grueso.

En Chad, más vale utilizar esła dosis, administrada de mayo a novien bre cuando el parasitismo gastro-intestinal del dromedario es el más importante. El thiabendazole es bastante poco toxico para el camello, excepto cuando los animales están atacados por otras enfermedades crónicas (Tripanosomiasis; abscesos varios, neumonias ete...). Entonces hay que reducir de mitad la dosis, pero en seguido volver a administrarla.

Después del tratamiento, el estado general rapidamente se mejora si el animal estâ sobre un buen pasto, mâs bien de tipo arbustiva. La ganancia de peso llega de 6,8 por 100 a 11 por 100.

Tendrâ que tomar algunas precauciones al utilizar el antihelmíntico en el camello.

\section{BIBLIOGRAPHIE}

BOUE (R.). - Essai de Barymétrie chez le dromadaire Nord-Africain. Rev. Elev. Med. Vét. Poys Trop., 1949, 3, I, 13-16.

FERRY (R.). - Parasitisme gastro-intestinal du dromadaire au Niger. Thèse vétérinaire, Paris, 1961, 46 pp.

GRABER (M.). - Rap. Tournée Nord-Ouaddai, Elev. Tchod, 1955, 127 pp.

GRABER (M.). - Etude dans certaines conditions africaines de l'action antiparasitaire du Thiabendazole sur divers Helminthes des animaux domestiques. I. Helminthes du zébu. Rev. Elev. Med. Vet. Pays Trop., $1965,18,1,39-58$.

GRABER (M.), TABO (R.) et SERVICE (J.).-Enquête sur les Helminthes du dromadaire tchadien. Etude des strongyloses gastrointestinales et de l'Haemoncose à Haemoncus longistipes. Rev. Elev. Med. Véf. Pays Trop. (à paraître en 1967).

GRABER (M.). - Renseignements concernant la biologie d'Haemonus longistipes du dromadaire étudiée dans des conditions expérimentales. Rev. Elev. Med. Vét. Pays trop., (à paraître en 1967).

IVASHKINA (E., E.). - Phenothiazine tested against Parabronemiasis of camels (CameIus bactrianus). Pap. Helm. Pres. Acad. Skjabin. Moscow Akad. Nouk, 1953, 254-257.

OZERSKAYA (V., N.). - Experimental application of Phénothiazine in Nematodiasis of the digestive tract of camels. Trud. Vseso. Inst. Gelmint. Imen. Akad. Skrjabina, 1953, 5, 165-166.

SPASSKI (A., A.). - Anoplocephalata Cestodes of domestic and wild animals. Principles of Cestodology. Moscou, 1951, I, 735.

STEWARD (J. S.). - Trichostrongylosis and Haemonchosis in the camel : their recognition and response to Phenothiazine. Vet. Rec., $1950,62,52,837-39$.

Rapport annuel secteur vétérinaire Ouaddaï Tchad, 1956, p. 24-25. 